\title{
Escala de Relacionamento da Díade: evidências de validade para cuidadores de idosos brasileiros
}

\author{
Francine Náthalie Ferraresi Rodrigues Queluz ${ }^{1}$ \\ Elizabeth Joan Barham ${ }^{2}$ \\ Lígia de Santis ${ }^{2}$ \\ Vanessa Santiago Ximenes ${ }^{2}$ \\ Acácia Aparecida Angeli dos Santos ${ }^{3}$ \\ ${ }^{1}$ Universidade São Francisco, SP, Brasil \\ ${ }^{2}$ Universidade Federal de São Carlos, SP, Brasil \\ ${ }^{3}$ Universidade São Francisco, SP, Brasil
}

\begin{abstract}
Resumo
A escala Dyadic Relationship Scale (DRS) foi traduzida para o Brasil com o nome de Escala de Relacionamento da Díade (ERD), visando avaliar a qualidade do relacionamento entre cuidadores e idosos dependentes. Este estudo teve como objetivo dar continuidade ao processo de obtenção de evidências de validade da ERD para o contexto brasileiro. Participaram 205 cuidadores de idosos familiares que responderam à ERD, ao Inventário de Depressão de Beck, à Escala de Burden de Zarit e à Escala de Qualidade de Vida. Por meio de uma análise fatorial confirmatória, observa-se que o modelo de dois fatores da versão estadunidense demonstrou bons índice de ajuste aos dados. A subescala "Interação Positiva" se relacionou positivamente com qualidade de vida e negativamente com depressão e sobrecarga. A subescala "Conflitos" se relacionou negativamente com qualidade de vida e positivamente com depressão e sobrecarga. Estas evidências da ERD continuam a apontar para sua validade.
\end{abstract}

Palavras-chave: Idosos; Cuidadores; Relacionamento Interpessoal; Qualidade de Vida; Avaliação Psicológica.

\section{Dyadic Relationship Scale: evidences of validity for Brazilian caregivers of elderly}

\begin{abstract}
The Dyadic Relationship Scale (DRS) was translated for use in Brazil as the Escala de Relacionamento da Díade (ERD), to evaluate the quality of the relationship between caregivers and their dependent relative. This study aimed to continue the process of obtaining evidence of validity for ERD in the Brazilian context. In this study, 205 caregivers of an elderly family member responded to the ERD, Beck Depression Inventory, Zarit Burden Interview, and Quality of Life Scale. Using confirmatory factor analysis, we observed satisfactory goodness of fit indices for the two-factor model that was reported for the United States version of this instrument. The subscale "Positive Interaction" was positively related to quality of life and negatively related to depression and burden. The "Conflicts" subscale was negatively related to quality of life and positively related to depression and burden. This evidence of the ERD continues to support its validity.
\end{abstract}

Keywords: Elderly; Caregivers; Interpersonal relationship; Quality of life; Psychological assessment.

\section{Escala de Relación de la Díada: evidencias de validez para cuidadores de ancianos brasileños}

\section{Resumen}

La Dyadic Relationship Scale (DRS) fue traducida a Brasil con el nombre de Escala de Relacionamento da Díade (ERD), para evaluar la calidad de relación entre cuidadores y ancianos dependientes. Este estudio tuvo como objetivo dar continuidad al proceso de obtención de evidencias de validez de la ERD para el contexto brasileño. Participaron 205 cuidadores de ancianos familiares que respondieron la ERD, al Inventario de Depresión de Beck, la Escala de Burden de Zarit y la Escala de Calidad de Vida. Por medio de un análisis factorial confirmatorio, se observa que el modelo de dos factores de la versión estadounidense demostró un buen índice de ajuste. La subescala "Interacción Positiva" se relacionó positivamente con calidad de vida y negativamente con depresión y sobrecarga. La subescala "Conflictos" se relacionó negativamente con calidad de vida y positivamente con depresión y sobrecarga. Estas evidencias acerca de la ERD siguen apuntando su validez.

Palabras clave: Adultos mayores; Cuidadores; Relacionamiento interpersonal; Calidad de vida; Evaluación Psicológica. 


\section{Introdução}

No atual cenário demográfico global no qual a população tem acesso a melhores condições médicosanitárias em conjunto com menores taxas de fecundidade, constata-se um aumento na expectativa de vida dos indivíduos e consequente elevação na parcela populacional de idosos (Kuchemann, 2012). Dado o aumento no número de anos vividos notase, também, uma acentuação no número de idosos dependentes devido ao surgimento de doenças crônicodegenerativas, como doenças coronárias, demências e outras (Kuchemann, 2012). Tal intensificação no grau de dependência do idoso gera a necessidade de assegurar a presença de cuidadores que o assistirão em suas atividades cotidianas e, dada a escassez de políticas públicas e o custo elevado de serviços particulares, a família se torna a provedora da maioria destes cuidados (Neri, 2014; Pinto \& Oliveira, 2015). Diante disto, percebe-se que entender como favorecer a qualidade da relação entre cuidadores e seus familiares idosos é de primordial importância para a qualidade de vida tanto de quem cuida, como de quem é cuidado.

Estudiosos revelam que o cuidador familiar é, em sua maioria, do sexo feminino, com idade média de 65 anos e alguém que não teve capacitação específica para ocupar esse papel (Del-Pino-Casado, MillánCobo, Palomino-Moral, \& Frías-Osuna, 2014; Neri, 2014) o que contribui para muitos desenvolverem uma percepção de sobrecarga moderada a severa, visto que essas pessoas se sentem despreparadas e desamparadas na atividade de cuidar (Caldeira, Neri, Batistoni, \& Cachioni, 2017; Neri, 2014; Pinto, 2016). O termo sobrecarga, conhecido internacionalmente como burden, refere-se a um sentimento produzido por contextos nos quais a percepção das demandas decorrentes das atividades de cuidar excede a capacidade de gerenciamento do cuidador, gerando estresse e ansiedade (Alvira et al. 2015; Del-PinoCasado et al., 2014). A falta de suporte familiar associada à renúncia de atividades próprias de trabalho e lazer podem gerar perdas financeiras e sociais, que contribuem, consequentemente, para uma maior sensação de sobrecarga por parte do cuidador e para que conflitos familiares ocorram (Alvira et al., 2015; Caldeira et al. 2017; Pinto, Barham, \& Del Prette, 2016). Isso pode fazer com que o cuidador apresente menor qualidade de vida quando comparado a não cuidadores após assumir a tarefa de cuidar um familiar idoso (Tomomitsu, Perracini, \& Neri, 2014)

Além da sobrecarga, a depressão é considerada outro desfecho negativo produzido pela atividade de cuidar de idosos. De acordo com o Manual Diagnóstico e Estatístico de Transtornos Mentais V (APA, 2014), a depressão é um distúrbio caracterizado pela presença de humor triste ou irritável, acompanhado de alterações somáticas e cognitivas que afetam significativamente a capacidade de funcionamento do indivíduo, implicando prejuízos intelectuais, sociais, afetivos, sexuais e profissionais. Ao refletir acerca da tarefa de cuidar de um idoso no contexto brasileiro, observa-se que tal atividade requer do cuidador dedicação praticamente exclusiva, o que pode gerar isolamento social, perda de atividades prazerosas, insônia e outras condições que contribuem para que o cuidador se torne mais propenso a apresentar sintomas depressivos, quando comparado a pessoas que não exercem a atividade de cuidar de idosos (Del-Pino-Casado et al., 2014; Tomomitsu et al., 2014).

Neri (2014) destaca que o cuidador exerce um papel basilar na qualidade de vida do idoso. Porém, a resposta emocional nesse contexto de cuidado, no qual há alta probabilidade de sobrecarga e depressão para o cuidador, gera sentimentos ambivalentes em relação ao idoso, comprometendo negativamente a percepção de qualidade de vida e a relação entre a díade cuidadoridoso (Dawood, 2016; Pinto, 2016; Tomomitsu et al., 2014). A este respeito, Pinto (2016) destaca que a relação entre o cuidador e seu familiar idoso pode ser enfraquecida por situações estressantes que surgem na convivência diária. Uma relação desgastada reduz a qualidade da assistência prestada ao idoso e eleva a probabilidade de ocorrência de abandono, negligência e violência contra o idoso enquanto, para o cuidador, intensifica a percepção de sobrecarga e estresse (Barham, Pinto, Andrade, Lorenzini, \& Ferreira, 2015; Pinto, Barham, \& Albuquerque, 2013).

No mesmo sentido, Sebern e Whitlatch (2007) ressaltaram a importância de considerar a influência mútua que existe entre os membros da díade cuidadoridoso como forma de compreender a prestação de cuidados. Isto é, existe uma acentuada semelhança em como cada membro da díade vivencia os efeitos de dar e receber cuidados. Assim, trocas interpessoais amenas, com equilíbrio e afeto entre o cuidador e o idoso, podem predizer menos sintomas depressivos, maior satisfação com a vida, e ainda maior facilidade no processo de adaptação à função de cuidar de alguém (Alvira et al., 2015; Caldeira et al. 2017; Ferreira, Queluz, Ximenes, Isaac, \& Barham, 2017; Lloyd, Patterson, \& Muers, 2014). Em contrapartida, relações diádicas conflituosas estão significantemente associadas à sintomatologia depressiva tanto do idoso, quanto do cuidador (Alvira et al., 2015; Lloyd et al., 2014; Sebern \& Whitlatch, 2007), deixando clara a função preditiva para uma melhor ou pior qualidade de 
vida da díade, a depender da forma como os membros se relacionam. Dessa maneira, estudos sobre a relação cuidador-idoso e formas de aferir essa relação podem subsidiar intervenções que promovam a redução de conflitos e a melhora na qualidade da relação da díade, de modo a tornar a convivência mais benéfica para ambos.

A relação entre cuidador e idoso pode ser resultante de um envolvimento diário baseado tanto em momentos de cooperação entre os membros da díade, quanto em trocas perniciosas, contribuindo para tensões entre o cuidador e o idoso. Poulshock e Deimling (1984) foram alguns dos primeiros pesquisadores a estudar as consequências destas diferentes formas de se relacionar em díades compostas por idosos dependentes e seus cuidadores. Estes autores investigaram a sobrecarga do cuidador, entendendo-a como uma variável interveniente que medeia a relação entre as demandas apresentadas pelo idoso e os impactos que a tarefa de cuidar tem sobre a vida de quem cuida. Eles utilizaram uma escala derivada da Zarit Burden Interview (Zarit, Orr, \& Zarit, 1985), com o objetivo de medir aspectos negativos da relação cuidador-idoso, para verificar quanto as tarefas de cuidado estão associadas a sentimentos de cansaço, dificuldades e aborrecimentos e quanto as condições do idoso são vistas como um problema para o cuidador. Os resultados mostraram que os prejuízos para a saúde física e mental do cuidador levaram a maior uso de drogas psicotrópicas, maior ocorrência de doenças psiquiátricas e maiores índices de estresse em cuidadores com piores relacionamentos interpessoais com o idoso cuidado. Resultados similares foram obtidos em pesquisas subsequentes (Loyd et al., 2014; Sebern \& Whitlatch, 2007)

Apesar de importantes, os estudos iniciais sobre o tema seguiram uma tendência internacional de analisar apenas aspectos negativos advindos da relação cuidador-idoso, ignorando possíveis benefícios dessa relação (Alvira et al., 2015; Loyd et al., 2014). Segundo Lloyd et al. (2014), a identificação de consequências positivas é importante por potencializar os benefícios do ato de cuidar, contribuindo para a manutenção e a qualidade da relação, como também por possibilitar a atuação de forma preventiva, evitando ou minimizando o surgimento de consequências negativas. Assim, diante da importância e do recente aumento no número de pesquisadores que investigam os aspectos positivos advindos da atividade de cuidar, sabe-se, hoje, que a relação cuidador-idoso, quando positiva, pode também predizer benefícios para vida do cuidador (Alvira et al., 2015; Ferreira et al., 2017; Loyd et al., 2014).

Além de alcançar mais informações sobre consequências positivas advindas da relação cuidador- idoso, outro avanço nessa área de estudos é a possibilidade de avaliar as consequências (positivas e negativas) da relação cuidador-idoso para a díade. Diante desta possibilidade, Sebern e Whitlatch (2007) adaptaram a medida utilizada por Poulshock e Deimling (1984), dando origem à Dyadic Relationship Scale (DRS). A DRS é uma medida estadunidense de avaliação da qualidade da relação da díade cuidadoridoso, que considera características positivas e negativas da relação na situação de cuidado, com duas versões: uma para cuidadores e outra para os idosos. No presente estudo, optou-se por obter informações sobre a validade da versão feita para o cuidador. Os itens deste instrumento (11 na versão do cuidador) consistem em afirmações a respeito da relação entre a díade e das transformações decorrentes da situação de cuidado. A estrutura interna da DRS, composta por dois fatores, foi estabelecida por meio de análises fatoriais exploratória e confirmatória. Os valores obtidos na análise fatorial confirmatória para o Comparative Fit Index (CFI) e para o Root Mean Square Error of Approximation (RMSEA) foram de 0,96 e 0,07, respectivamente. Os autores optaram por tratar os fatores como duas subescalas, por serem construtos distintos, gerando dois escores para cada fator e não um total para a escala. Assim, o instrumento inclui uma subescala para medir a interação positiva entre cuidador-idoso e outra para medir os conflitos entre os membros da díade. Quanto maior a pontuação em cada subescala, maior é o grau de interação positiva ou de conflitos entre os membros da díade. Por meio de análises de regressão foi evidenciada a validade concorrente da subescala de conflitos da DRS com um indicador de depressão. Adicionalmente, os autores também verificaram evidências de validade convergente e discriminante para as duas dimensões da DRS, nas versões para o cuidador e para o idoso, por meio de técnicas de variância extraída da média.

No contexto, brasileiro nota-se a falta de medidas com parâmetros psicométricos adequados que possam ser usadas para avaliar a qualidade da relação da díade idoso-cuidador, de forma que as poucas ferramentas existentes ainda carecem de estudos de validade que deem sustentação às interpretações por elas obtidas. Com instrumentos mais confiáveis, será possível avaliar esforços para promover interações mais positivas e menos conflituosas entre cuidador e idoso, já que este envolvimento é cada dia mais prevalente no país. Ao considerar a fundamentação teórica da DRS, condizente com as necessidades da área e a adequação de suas características psicométricas, Thomazatti e Barham (2010) deram início ao processo de obtenção 
de evidências de validade da DRS para o Brasil. As autoras traduziram e adaptaram a escala, dando origem à Escala de Relacionamento da Díade (ERD). Antes de ser aplicada em idosos e cuidadores, a ERD passou pelo crivo de juízes especialistas na área de psicologia, que atestaram a validade semântica e de conteúdo do instrumento para o contexto brasileiro e também foi realizado um estudo piloto.

Diante da importância de obter uma medida como a ERD que apresente evidências de validade para o uso no Brasil e que tenha características psicométricas equivalentes às da escala nos Estados Unidos, o objetivo deste estudo foi dar continuidade ao processo de obtenção de evidências de validade da ERD, versão do cuidador, para o contexto brasileiro. Neste estudo serão investigadas evidências de validade interna, em relação à sua estrutura interna e as estimativas de precisão do instrumento (estrutura fatorial e confiabilidade interna) e validade de construto do tipo convergente (relação com variáveis correlatas - qualidade de vida, depressão e sobrecarga do cuidador) (AERA, APA, \& NMCE, 2014).

Esperava-se que escores na subescala de interação positiva se relacionassem positivamente com indicadores de qualidade de vida e negativamente com indicadores de depressão e sobrecarga do cuidador. De forma análoga, esperava-se que escores na subescala de conflitos se relacionassem negativamente com indicadores de qualidade de vida e positivamente com indicadores de depressão e sobrecarga do cuidador.

\section{Método}

\section{Participantes}

Participaram do estudo 205 cuidadores de idosos familiares, com idade média de 51 anos $(\mathrm{DP}=14,4)$, variando entre 18 e 87 anos. Em média, eles exerciam essa tarefa há cinco anos $(\mathrm{DP}=4,7)$, variando entre 6 meses e 20 anos. Os cuidadores foram recrutados junto ao Serviço de Atendimento Domiciliar (SAD) das cidades de São Carlos e de Campinas, na Unidade Saúde Escola da Universidade Federal de São Carlos e por indicação de outros participantes ou de pessoas conhecidas pelas pesquisadoras, nas cidades de São Paulo e de Itapira, todas no estado de São Paulo. Como critério de inclusão, teriam que ser maiores de 18 anos e familiares dos idosos que cuidavam. Cuidadores formais ou pagos foram excluídos desta amostra. A coleta de dados foi realizada nas residências dos mesmos ou em algum lugar escolhido por eles, que garantisse o sigilo necessário. Os dados sociodemográficos dos cuidadores são apresentados na Tabela 1.
TABELA 1

Perfil Sociodemográfico dos Participantes $(N=205)$

\begin{tabular}{|c|c|c|}
\hline Variável & $n$ & $\%$ \\
\hline \multicolumn{3}{|l|}{ Sexo } \\
\hline Feminino & 180 & 87,8 \\
\hline Masculino & 25 & 12,2 \\
\hline \multicolumn{3}{|l|}{ Faixa etária (anos) } \\
\hline $18-30$ & 24 & 11,7 \\
\hline $31-50$ & 58 & 28,3 \\
\hline $51-70$ & 109 & 53,2 \\
\hline Acima 70 & 14 & 6,8 \\
\hline \multicolumn{3}{|l|}{ Estado Civil } \\
\hline Solteiro & 59 & 28,8 \\
\hline Casado/ União estável & 105 & 51,2 \\
\hline Separado & 27 & 13,2 \\
\hline Viúvo & 14 & 6,8 \\
\hline \multicolumn{3}{|l|}{ Escolaridade } \\
\hline Analfabeto ou Primário incompleto & 19 & 9,3 \\
\hline Primário ou Ginásio incompleto & 36 & 17,6 \\
\hline Ginásio ou Colegial incompleto & 21 & 10,1 \\
\hline Colegial ou Superior incompleto & 53 & 25,9 \\
\hline Superior completo & 76 & 37,1 \\
\hline \multicolumn{3}{|l|}{ Classe Social } \\
\hline A2 & 16 & 7,8 \\
\hline $\mathrm{B} 1 / \mathrm{B} 2$ & 67 & 32,7 \\
\hline $\mathrm{C} 1 / \mathrm{C} 2$ & 98 & 47,8 \\
\hline $\mathrm{D}$ & 24 & 11,7 \\
\hline \multicolumn{3}{|l|}{ Relação com o idoso } \\
\hline Filha(o) & 138 & 67,3 \\
\hline $\operatorname{Neta}(0)$ & 21 & 10,2 \\
\hline Cônjuge & 19 & 9,3 \\
\hline Nora/genro & 15 & 7,3 \\
\hline Irmã/Irmão & 9 & 4,4 \\
\hline Outro & 3 & 1,5 \\
\hline \multicolumn{3}{|l|}{ Cidade } \\
\hline Pequeno Porte & 34 & 16,6 \\
\hline Médio Porte & 118 & 57,6 \\
\hline Metrópole & 53 & 25,8 \\
\hline
\end{tabular}

\section{Instrumentos}

Características Sociodemográficas. Um questionário foi elaborado para verificar as características sociodemográficas (sexo, idade, escolaridade, estado civil, entre outras) dos participantes, visando a descrever a amostra obtida.

Critério de Classificação Econômica Brasil (ABEP, 2013). Este instrumento permite avaliar o nível socioeconômico das famílias com base no poder aquisitivo, no número de bens de consumo duráveis, na presença de empregada doméstica e no grau de instrução do chefe da família, dividindo a população em sete classes (A, B1, B2, C1, C2, D e E).

Escala de Relacionamento da Díade (ERD). Esta escala é uma versão em português da Dyadic 
Relationship Scale (Sebern \& Whitlatch, 2007), usada para examinar a qualidade da relação cuidador-idoso, levando em consideração seus aspectos negativos e positivos. Há uma versão específica para cada membro da díade. Na versão do cuidador, o instrumento é composto por 11 afirmações a respeito da qualidade da relação entre os membros da díade e das transformações decorrentes da situação de cuidado. Para cada afirmação, há quatro opções de resposta: discordo plenamente (1 ponto), discordo ( 2 pontos), concordo (3 pontos) ou concordo plenamente (4 pontos). A Dyadic Relationship Scale é composta por duas subescalas: uma que mede a "Interação positiva" e a outra que mede os "Conflitos" entre os membros da díade cuidador idoso. Quanto maior a pontuação nas duas subescalas, maior é o grau de interação positiva (Subescala 1) ou de conflitos (Subescala 2). A versão estadunidense da escala apresenta $\alpha=0,89$ para a subescala "Conflitos" e $\alpha=0,85$ para a subescala "Interação positiva".

Inventário de Depressão de Beck (BDI). O BDI (Beck Depression Inventory de Beck, Rush, Shaw, \& Emery, 1979) é aplicado para detectar sintomas e nível de depressão. Trata-se de uma escala de autorrelato, composta por 21 itens, sendo que cada item tem quatro afirmações graduadas de menor ( 0 pontos) a maior (3 pontos) severidade de sintomas depressivos. O BDI é formado por três fatores: autodepreciação $(\alpha=0,76)$, cognição-afeto $(\alpha=0,77)$ e somática $(\alpha=0,66)$. Escores entre 0 e 11 pontos indicam ausência de depressão; entre 12 e 19 pontos indicam depressão leve; entre 20 e 35 pontos indicam depressão moderada; e acima de 36 pontos indicam depressão grave. No estudo de referência para o português, o inventário apresentou consistência interna de $\alpha=0,81$ (Gorenstein \& Andrade, 1998).

Escala de Burden de Zarit (ZBI) (Zarit et al., 1985). O nível de sobrecarga dos cuidadores foi medido por meio da Escala de Burden de Zarit, adaptado para uso no Brasil por Taub, Andreoli e Bertolucci (2004). A escala é composta por 22 questões que avaliam a percepção de sobrecarga por parte de cuidadores familiares de idosos. As alternativas de resposta para cada item são: nunca ( 0 pontos), raramente (1 ponto), algumas vezes ( 2 pontos), muito frequentemente ( 3 pontos) ou sempre (4 pontos). A pontuação final pode variar entre 0 e 88 pontos. A versão brasileira da ZBI é avaliada com base na pontuação total do participante e a severidade da sobrecarga é estabelecida da seguinte maneira: menos de 21 pontos, ausência de sobrecarga ou sobrecarga mínima; 21 a 40 pontos, sobrecarga leve a moderada; 41 a 60 pontos, sobrecarga moderada a severa; 61 a 88 pontos, sobrecarga severa. A escala apresenta quatro fatores descritos na literatura internacional e, no Brasil, por Taub et al. (2004): "Impacto da prestação de cuidados", "Relação interpessoal", "Expectativas com o cuidar" e "Percepção de autoeficácia". O instrumento também pode ser considerado unidimensionalmente, apresentando uma consistência interna global de $\alpha=0,87$ (Scazufca, 2002).

Escala de Qualidade de Vida (QdV-DA) (Logsdon, Gibbons, McCurry, \& Teri, 1999). A escala foi validada para uso no Brasil por Novelli (2006) e é um instrumento que pode ser usado para analisar a percepção de qualidade de vida por parte de cuidadores de idosos, usando uma linguagem simples que pode ser entendido também por idosos com doença de Alzheimer leve a moderada. $\mathrm{O}$ instrumento é composto de 13 itens (por exemplo, saúde, casamento, moradia, etc.), quantificados em uma escala de quatro pontos, com a pontuação 1 sendo atribuída à qualificação ruim e a pontuação 4, excelente. A pontuação total varia entre 13 e 52, com pontuações mais altas indicando maior qualidade de vida. A versão brasileira desta escala é unidimensional e tem uma boa consistência interna, avaliada por meio do alfa de Cronbach $(\alpha=0,86)$.

\section{Procedimento de coleta de dados}

Inicialmente, foi feito contato com representantes da Secretaria de Saúde da Prefeitura Municipal de São Carlos e de Campinas e com os Serviços de Atendimento Domiciliar destas cidades. Os cuidadores que se enquadraram dentro dos critérios foram convidados a participar da pesquisa. Após a obtenção da autorização, foi agendado um horário para a aplicação dos instrumentos, de modo a garantir sigilo e privacidade. Ao encontrar cada participante, o Termo de Consentimento Livre e Esclarecido foi lido em voz alta e sanadas eventuais dúvidas e depois, foi realizada a aplicação dos instrumentos.

\section{Procedimento de análise de dados}

Para verificar a distribuição dos escores para cada instrumento, foram calculados a média, o desvio padrão, os valores mínimos e máximos, além de indicadores de curtose e assimetria para cada variável. Tendo em vista que a coleta de dados foi realizada por meio de entrevistas individuais, não houve problemas com dados omissos. Todas as variáveis apresentaram uma distribuição normal, segundo a inspeção dos valores de modas, de curtose e assimetria e do teste de normalidade de Kolmogorov-Smirnov (Marôco, 2014; Pasquali, 2015). Assim, para confirmar a estrutura traduzida anteriormente por Thomazatti e Barham (2010), além de medir sua consistência interna, foram realizadas análises fatoriais confirmatórias e o cálculo do alfa de Cronbach no programa MPlus 7. Os índices 
de ajuste considerados foram: Comparative Fit Index (CFI $\geq 0,90)$, Root Mean Square Error of Approximation (RMSEA, $\leq 0,06$ ou $\leq 0,08$ com intervalo de confiança de 90\%), a significância estatística do teste de Quiquadrado $(\mathrm{p} \leq 0,05)$ e o Qui-quadrado dividido pelo grau de liberdade $\left(\chi^{2} / \mathrm{gl}<3\right)$ ( Hu \& Bentler, 1999). Para verificar possíveis correlações entre as variáveis, foi utilizado o coeficiente de Correlação de Pearson, uma vez que a amostra apresentou distribuição normal. Para o presente estudo, a magnitude das correlações foi classificada em: fraca $(<0,30)$, moderada $(0,30$ a $0,59)$, forte $(0,60$ a 0,69$)$, muito forte $(0,70$ a 0,99$)$, ou perfeita $(1,0)$ (Levin \& Fox, 2004). Os dados de correlação foram analisados com o software SPSS Statistics, versão 20.

\section{Procedimentos éticos}

O projeto foi aprovado pelo Comitê de Ética da Universidade Federal de São Carlos (Parecer $\mathrm{n}^{\mathrm{o}}$ 144.507/2012). Todos os participantes receberam informações acerca dos objetivos da pesquisa, das atividades a serem desenvolvidas e de seus direitos, antes de assinarem o Termo de Consentimento Livre e Esclarecido. A identidade dos participantes foi mantida em sigilo.

\section{Resultados}

\section{Estrutura interna e precisão do instrumento}

Na Tabela 2, é apresentada a comparação entre os diferentes modelos propostos para a Escala de Relacionamento da Díade que foram calculados por meio da análise fatorial confirmatória. Foram comparados os modelos: (1) dois fatores com rotação oblíqua, (2) dois fatores com rotação ortogonal, (3) unifatorial e (4) bi-factor. De acordo com os resultados observados na Tabela 2, percebe-se que todos os modelos apresentaram um valor de $\mathrm{x}^{2}$ significativo, além disso os modelos que mostraram o $\mathrm{x}^{2} / \mathrm{gl}$ mais adequados foram os modelos de dois fatores com rotação oblíqua, dois fatores com rotação ortogonal e o Bi-factor, sendo que o melhor valor apresentado foi pelo modelo de dois fatores com rotação oblíqua. No que diz respeito ao CFI, os mais adequados foram os modelos de dois fatores com rotação oblíqua e o Bi-factor, ambos apresentaram um valor de 0,90, considerado satisfatório dentro da teoria clássica dos itens (Hu \& Bentler, 1999). No que diz respeito ao valor do RMSEA, o modelo mais adequado foi o de dois fatores com rotação oblíqua. Portanto, ao comparar os quatro modelos e levando em consideração os índices de ajustes, o melhor modelo foi o de rotação oblíqua com dois fatores. Este modelo foi o mesmo proposto por Sebern e Whitlatch (2007) na versão estadunidense da escala e é o que serviu de base para a tradução e adaptação do instrumento para o contexto brasileiro, realizada por Thomazatti e Barham (2010).

TABELA 2

Comparação entre Diferentes Modelos para a

Escala de Relacionamento da Díade e seus Respectivos Índices de Ajustes

\begin{tabular}{lcccc}
\hline Modelo & $\chi^{2} / g l$ & $p$ & $C F I$ & $R M S E A$ \\
Valor de referência & $<3$ & $\leq 0,05$ & $>0.90$ & $<0.08$ \\
Dois fatores oblíquo & 2,27 & 0,001 & 0,90 & 0,08 \\
Dois fatores ortogonal & 2,53 & 0,001 & 0,88 & 0,09 \\
Unifatorial & 5,98 & 0,001 & 0,60 & 0,16 \\
Bi-factor & 2,45 & 0,001 & 0,90 & 0,09 \\
\hline
\end{tabular}

Como o modelo que se mostrou mais adequado foi o de dois fatores com rotação oblíqua, foram também calculadas as cargas fatoriais e os valores de precisão para as duas subescalas dentro deste modelo. Os resultados estão apresentados na Tabela 3.

TABELA 3

Itens e Cargas Fatoriais da Escala de Relacionamento da Díade

\begin{tabular}{ccc}
\hline Item/Cargas fatoriais & Conflitos & Interação Positiva \\
9 & 0,87 & \\
10 & 0,84 & \\
8 & 0,82 & \\
7 & 0,76 & \\
11 & 0,46 & \\
2 & & 0,75 \\
1 & & 0,73 \\
6 & & 0,73 \\
3 & & 0,61 \\
5 & & 0,61 \\
4 & & 0,58 \\
\hline
\end{tabular}

Todos os valores de cargas fatoriais foram considerados aceitáveis, sendo maiores que 0,40 (Hair, Anderson, Tatham, \& Black, 2005). Para a subescala de "Conflitos" e de "Interação Positiva", a consistência interna foi de 0,81 e 0,77 , respectivamente. Ambos os valores são considerados valores de precisão adequados (Marôco, 2014).

\section{Evidências de validade de construto do tipo convergente}

Por meio da prova de Pearson, foi calculado o índice das correlações entre os fatores da Escala de Relacionamento da Díade com os demais instrumentos, 
a saber, BDI, ZBI e QdV-DA. Os resultados são apresentados na Tabela 4.

\section{TABELA 4}

Correlações entre Escores da Escala de Relacionamento da Díade e Escores em Medidas de Construtos Relacionados

\begin{tabular}{lcc}
\hline Variáveis externas & $\begin{array}{c}\text { Interação } \\
\text { Positiva }\end{array}$ & Conflitos \\
Qualidade de Vida & $0,29^{*}$ & $-0,44^{*}$ \\
Depressão & $-0,20^{*}$ & $0,48^{*}$ \\
F1 - Autodepreciação & $-0,15^{*}$ & $0,32^{*}$ \\
F2 - Cognição/Afeto & $-0,21^{*}$ & $0,42^{*}$ \\
F3 - Dimensão Somática & $-0,16^{*}$ & $0,42^{*}$ \\
Sobrecarga Total & $-0,36^{*}$ & $0,63^{*}$ \\
F1 - Impacto da prestação de cuidados & $-0,29^{*}$ & $0,59^{*}$ \\
F2 - Impacto na relação interpessoal & $-0,43^{*}$ & $0,55^{*}$ \\
F3 - Expectativas com o cuidar & $-0,33^{*}$ & $0,48^{*}$ \\
F4 - Percepção de auto eficácia & 0,08 & 0,02 \\
\hline
\end{tabular}

$* p<0,05$; os valores apresentados na tabela referem-se aos valores de correlação de Pearson $(r)$.

De acordo com os resultados apresentados na Tabela 4, percebe-se que a subescala de Interação Positiva se relacionou positivamente com qualidade de vida e negativamente com depressão e seus respectivos fatores, assim como com a sobrecarga total e os escores nos fatores Impacto da prestação de cuidados, Relação interpessoal e Expectativas com o cuidar do ZBI. Já a subescala de Conflitos se relacionou negativamente com qualidade de vida e positivamente com depressão e seus respectivos fatores, e também com sobrecarga total e com os fatores Impacto da prestação de cuidados, Relação interpessoal e Expectativas com o cuidar do ZBI. As correlações entre Interação Positiva e qualidade de vida, depressão e seus fatores foram de magnitude fraca. Já as correlações entre Interação Positiva e sobrecarga total e os fatores Relação interpessoal e Expectativas com o cuidar do ZBI foram de magnitude moderada, enquanto a correlação com o fator Impacto da prestação de cuidados foi de magnitude fraca.

\section{Discussão}

O objetivo do presente estudo foi dar continuidade ao processo de obtenção de evidências de validade da ERD, versão do cuidador, para o contexto brasileiro. Os dados obtidos permitem afirmar que foram encontradas evidências de validade para a ERD, além de valores de precisão adequados. Em termos de estrutura interna, os resultados obtidos no Brasil vão ao encontro da versão estadunidense do instrumento, pois a estrutura com dois fatores correlacionados se confirmou e apresentou valores similares. Por exemplo, na versão estadunidense, os valores encontrados para os índices de referência foram: $\mathrm{CFI}=0,96, \mathrm{RMSEA}=0,07 \mathrm{e}$ $\chi^{2} / \mathrm{gl}=2,10$ (Sebern \& Whitlatch, 2007), e na versão brasileira, foram: $\mathrm{CFI}=0,90, \mathrm{RMSEA}=0,08 \mathrm{e}$ $\chi^{2} / \mathrm{gl}=2,27$ - valores considerados adequados para garantir a validade de uma escala e que são muito próximos, na comparação entre os dois países (Marôco, 2014; Pasquali, 2015). Portanto, pode-se afirmar que em comparação com a estrutura interna da versão estadunidense do instrumento, a estrutura confirmada para a versão brasileira (ERD) e estadunidense (Sebern \& Whitlatch, 2007) (DRS) da escala são similares. Assim, além do respaldo teórico, a confirmação de estruturas internas similares, em países diferentes, possibilita a comparação fiel da relação estabelecida por cuidadores de idosos de diferentes culturas.

Em relação à correlação de escores da ERD e das variáveis externas avaliadas neste estudo, nota-se que os resultados encontrados confirmaram as hipóteses iniciais, evidenciando a validade de construto do tipo convergente da ERD, versão do cuidador, quando aplicada no Brasil. Neste sentido, a subescala Interação positiva apresentou correlação positiva, apesar de fraca, com o indicador de qualidade de vida dos cuidadores. Ainda como esperado, esta subescala se correlacionou negativamente com os indicadores de depressão (todos de magnitude fraca) e de sobrecarga do cuidador (as magnitudes das correlações variaram entre fraca e moderada). Ou seja, pode-se afirmar que de maneira geral um cuidador que tem mais interação positiva com o idoso que cuida, tem uma melhor qualidade de vida e menor probabilidade de apresentar depressão e sobrecarga. Estes resultados são condizentes com os apresentados por diversos pesquisadores (Alvira et al. 2015; Ferreira et al., 2017; Loyd et al., 2014; Pinto \& Barham, 2014; Pinto et al., 2016; Pinto \& Oliveira, 2015) que encontraram evidências da relação entre aspectos positivos da atividade de ser um cuidador e menores níveis de depressão e sobrecarga, assim como melhores níveis de qualidade de vida em cuidadores de idosos quando os mesmos têm uma melhor relação com o idoso cuidado. $\mathrm{O}$ único indicador que não apresentou correlação estatisticamente significativa com a subescala Interação Positiva da ERD foi o fator 4 do instrumento de sobrecarga, que diz respeito à Percepção de Auto eficácia. Esse resultado pode ser explicado pelo fato de que os cuidadores podem sentir que prestam assistência de qualidade a seu parente idoso independente da qualidade de sua interação. Essa qualidade pode estar ligada às possibilidades de cada um, por exemplo, mesmo não apresentando uma interação positiva com o idoso, o cuidador faz tudo o 
que pode para que o idoso fique bem, de acordo com o que o cuidador tem a oferecer.

Analogamente e também corroborando as hipóteses levantadas, a subescala de Conflitos se correlacionou negativamente com o indicador de qualidade de vida do cuidador, sendo esta uma correlação moderada. Também, o escore nesta subescala apresentou correlação positiva e moderada com os indicadores de depressão e uma correlação forte com o escore de sobrecarga total do cuidador e moderada com os fatores 1,2 e 3 do instrumento de sobrecarga. Pode-se afirmar que, de maneira geral, um cuidador que tem mais conflitos com o idoso que cuida, tem uma pior qualidade de vida e maior probabilidade de apresentar depressão e sobrecarga. Estes resultados são condizentes com os apresentados por diversos pesquisadores (Alvira et al. 2015; Loyd et al., 2014; Pinto \& Barham, 2014; Pinto et al., 2016; Pinto \& Oliveira, 2015; Sebern \& Whitlatch, 2007). A relação entre o fator de Conflitos da versão estadunidense da escala e um indicador de depressão também foi avaliada por Sebern e Whitlatch (2007). As autoras encontraram um coeficiente de regressão, entre as medidas, de 0,47 $(\mathrm{p}<0,05)$. Este resultado está muito próximo ao verificado no presente estudo que, ao correlacionar a subescala de Conflitos com escores no BDI, verificou uma correlação de $0,48(\mathrm{p}<0,001)$. Apesar de utilizarem diferentes instrumentos para avaliarem sintomas depressivos, os resultados apresentados se confirmam, fortalecendo as evidências de validade da ERD. No entanto, como no fator Interação Positiva da ERD, o único indicador que não apresentou correlação estatisticamente significativa com Conflitos foi o fator 4 do instrumento de sobrecarga, que diz respeito à Percepção de Auto eficácia. A explicação para a falta de correlação é parecida com a anterior, já que um cuidador pode ter conflitos com o idoso que ele cuida, mas acreditar que, mesmo assim, faz o melhor que pode dentro de suas possibilidades, não afetando então, sua percepção de auto eficácia como cuidador (Pinto \& Barham, 2014).

Ademais, com a tendência internacional de destacar não apenas os aspectos negativos consequentes da relação cuidador-idoso para o cuidador, mas também se atentar aos possíveis benefícios advindos desta relação (Alvira et al., 2015; Lloyd et al., 2014), a escala traduzida pode servir como forma de aferir intervenções que se voltem para a diminuição de conflitos na díade e ao aumento do número de interações positivas durante o exercício da tarefa de cuidar de um familiar idoso dependente, assim como para a produção de novos conhecimentos sobre o tema. Neste sentido, a confirmação de uma estrutura interna composta pelas subescalas de Conflito e Interação positiva possibilita que ambos os aspectos (negativo e positivo) sejam mensurados.

Para se contar com uma medida com evidências de validade para o Brasil para mensurar a qualidade da relação da díade cuidador-idoso, segundo a percepção do cuidador, ainda são necessários estudos adicionais sobre o tema. Assim, é preciso que a estrutura interna da ERD apresentada no presente estudo seja confirmada com uma segunda amostra de cuidadores brasileiros que sejam de outros estados (além do estado de São Paulo), representando, ao menos, as cinco grandes regiões do país, sendo esta uma limitação do presente estudo. Outra limitação foi o número pequeno de cuidadores do sexo masculino. No entanto, esse número baixo de cuidadores homens está de acordo com os dados da literatura (Alvira et al. 2015) que mostram que as mulheres são as principais cuidadoras, em números, de seus parentes idosos nas famílias.

A fim de confirmar as correlações verificadas entre a qualidade da relação da díade e os demais construtos analisados (qualidade de vida, depressão e sobrecarga), escores na ERD poderiam ser relacionados com diferentes indicadores destes correlatos - outras escalas ou observações, além da verificação de outros construtos, como presença de apoio social, dependência funcional do idoso, estresse, ansiedade, entre outros. Além disso, um instrumento que avaliasse se a EDR apresenta desejabilidade social também seria interessante, como forma de verificar se os cuidadores apresentam esse viés de resposta (Marocô, 2014; Pasquali, 2015). Dados adicionais sobre a estrutura interna e a validade externa da ERD, como os mencionados, endossariam um corpo de evidências importantes para que fosse feita, futuramente, a normatização da escala, para o Brasil. Por fim, no presente estudo, optou-se por verificar apenas se a versão do cuidador da ERD apresentava evidências de validade. Em estudos futuros, também seria importante verificar as evidências de validade para a versão do idoso. Isso faria com que os dois participantes da díade (cuidador e idoso) pudessem ser comparados em relação à sua percepção da qualidade do relacionamento no qual estão inseridos. A verificação da qualidade psicométrica do instrumento de avaliação da relação da díade, também para o idoso brasileiro, contemplará mais esta lacuna na área, contribuindo para o seu avanço. 


\section{Referências}

ABEP (2013). Critério de Classificação Econômica Brasil. Associação Brasileira de Empresas de Pesquisa.

Alvira et al. (2015). The association between positive-negative reactions of informal caregivers of people with dementia and health outcomes in eight European countries: A cross-sectional study. Journal of Advanced Nursing, 71(6), 1417-1434. https://doi.org/10.1111/jan.12528

American Educational Research Association, American Psychological Association, \& National Council on Measurement in Education (2014). Standards for educational and psychological testing. Washington, DC: American Educational Research Association.

American Psychiatric Association. (2014). DSM-5: Manual diagnóstico e estatístico de transtornos mentais. Artmed Editora.

Barham, E. J., Pinto, F. N. F. R., Andrade, A. R., Lorenzini, M. F. J., \& Ferreira, C. R. (2015). Fundamentos e estratégias de intervenção para a promoção de saúde mental em cuidadores de idosos. In: S. G. Murta, C. Leandro-França, K. B. Santos, \& L. Polejack, (Orgs.). Prevenção e promoção em saúde mental: Fundamentos, planejamento e estratégias de intervenção (pp. 844-862). Novo Hamburgo, RS: Sinopsys.

Beck, A. T., Rush, A. J., Shaw, B.F., \& Emery, G. (1979). Cognitive therapy of depression: A treatment manual. New York: Guilford Press.

Caldeira, R. B., Neri, A. L., Batistoni, S. S. T., \& Cachioni, M. (2017). Variáveis associadas à satisfação com a vida em cuidadores idosos de parentes também idosos cronicamente doentes e dependentes. Revista Brasileira de Geriatria e Gerontologia, 20(4), 502-515. https://doi.org/10.1590/1981-22562017020.160177

Dawood, S. (2016). Caregiver Burden, Quality of Life and vulnerability towards psychopathology in Caregivers of Patients with Dementia/Alzheimer's Disease. Journal of the College Physicians Surgeons Pakistan, 26(11), 892-895.

Del-Pino-Casado, R., Millán-Cobo, M. D., Palomino-Moral, P. A., \& Frías-Osuna, A. (2014). Cultural correlates of burden in primary caregivers of older relatives: A Cross-sectional study. Journal of Nursing Scholarship, 46(3), 176-186. https://doi.org/10.1111/jnu.12070

Ferreira, C. R., Queluz, F. N. F. R., Ximenes, V. S., Isaac, L., \& Barham, E. (2017). P3Es e a diminuição da sobrecarga em cuidadores: Confirmando efeitos em curto e longo prazo. Revista Kairós - Gerontologia, 20(3), 131-150. https:// doi.org/10.23925/2176-901X.2017v20i3p131-150

Gorenstein, C. \& Andrade, L. H. S. G. (1998). Inventario de depressão de Beck: Propriedades psicométricas da versão em português. Revista de Psiquiatria Clínica, 25(5), 245-250.

Hair, J. F., Anderson, R. E., Tatham, R. L., \& Black, W. C. (2005). Análise multivariada de dados. In: A. S. Sant'Anna, \& A. C. Neto (Trans). Porto Alegre, RS: Bookman.

Hu, L. \& Bentler, P. M. (1999). Cutoff criteria for fit indexes in covariance structure analysis: Conventional criteria versus new alternatives. Structural Equation Modeling: A Multidisciplinary Journal, 6(1), 1-55. https://doi. org/10.1080/10705519909540118

Küchemann, B. A. (2012). Envelhecimento populacional, cuidado e cidadania: Velhos dilemas e novos desafios. Sociedade e Estado, 27(1), 165-180. https://doi.org/10.1590/S0102-69922012000100010

Levin, J. \& Fox, J. A. (2004). Estatística para ciências humanas. São Paulo, SP: Pearson.

Lloyd, J., Patterson, T., \& Muers, J. (2014). The positive aspects of caregiving in dementia: A critical review of the qualitative literature. Dementia, O(0), 1-28. https://doi.org/10.1177/1471301214564792

Logsdon, R. G., Gibbons, L. E., McCurry, S. M., \& Teri, L. (1999). Quality in life in Alzheimer's disease: Patient and Caregiver Reports. Journal of Mental Health Aging, 1(5), 21-32.

Marôco, J. (2014). Análise estatística com o SPSS Statistics. Pêro Pinheiro, Portugal: Report Number.

Neri, A. L. (2014). Palavras-chave em gerontologia. Campinas, SP: Editora Alínea.

Novelli, M. M. P. C. (2006). Validação da escala de qualidade de vida (QdV-DA) para pacientes com doença de Alzheimer e seus respectivos cuidadores familiares. Tese de doutorado, Programa de Pós-Graduação em Medicina, Universidade de São Paulo, São Paulo. https://doi.org/10.11606/T.5.2006.tde-17102014-120122

Pasquali, L. (2015). Delineamento de pesquisa em ciência (Vol. 2). São Paulo, SP: Vetor.

Pinto, F. N. F. R. \& Barham, E. J. (2014). Habilidades sociais e estratégias de enfrentamento de estresse: Relação com indicadores de bem-estar psicológico em cuidadores de idosos de alta dependência. Revista Brasileira de Geriatria e Gerontologia, 15(3), 525-539. https://doi.org/10.1590/1809- 9823.2014.13043

Pinto, F. N. F. R. \& Oliveira, D. C. (2015). Capacidade funcional e envolvimento social em idosos: Há relação? Revista Brasileira de Ciências do Envelhecimento Humano, 12(1), 56-68. https://doi.org/10.5335/rbceh.v12i1.4687

Pinto, F. N. F. R. (2016). Violência contra o idoso: Uma discussão sobre o papel do cuidador. Revista Kairós Gerontologia, 19(2), 107-119.

Pinto, F. N. F. R., Barham, E. J., \& Del Prette, Z. A. P. (2016). Interpersonal conflicts among family caregivers of the elderly: The importance of social skills. Paidéia (Ribeirão Preto), 26(64), 161-170. https://doi.org/10.1590/198243272664201605

Poulshock, S. W. \& Deimling, G. T. (1984). Families Caring for Elders in Residence: Issues in the Measurement of Burden. Journal of Gerontology, 39(2), 230-239. https://doi.org/10.1093/geronj/39.2.230 
Scazufca, M. (2002). Versão brasileira da escala Burden Interview para avaliação de sobrecarga em cuidadores de indivíduos com doenças mentais. Revista Brasileira de Psiquiatria, 24(1), 12-17. https://doi.org/10.1590/S151644462002000100006

Sebern, M. D. \& Whitlatch, C. J. (2007). Dyadic relationship scale: A measure of the impact of the provision and receipt of family care. The Gerontologist, 47(6), 741-751. https://doi.org/10.1093/geront/47.6.741

Shafer, A. B. (2006). Meta-analysis of the Factor Structures of Four Depression Questionnaires: Beck, CES-D, Hamilton, and Zung. Journal of clinical psychology, 62(1), 123-146. https://doi.org/10.1002/jclp.20213

Taub, A., Andreoli, S. B., \& Bertolucci, P. H. (2004). Dementia caregiver burden: Reliability of the brazilian version of the Zarit caregiver burden interview. Caderno de Saúde Pública, 20(2), 372-376. https://doi.org/10.1590/S0102$311 X 2004000200004$

Thomazatti, A. P. G. \& Barham, E. J. (2010). Integrando medidas qualitativas e quantitativas para avaliar a qualidade do relacionamento mãe-idosa e filha-cuidadora. Trabalho apresentado no XVII Congresso de Iniciação Científica, Universidade Federal de São Carlos, São Carlos, SP.

Tomomitsu, M. R. S. V., Perracini, M. R., \& Neri, A. L. (2014). Fatores associados à satisfação com a vida em idosos cuidadores e não cuidadores. Ciência \& Saúde Coletiva, 19(8), 3429-3440. https://doi.org/10.1590/141381232014198.13952013

Zarit, S. H., Orr, N. K., \& Zarit J. M. (1985). The hidden victims of Alzheimer's disease: Families under stress. New York: New York University Press.

Dados dos autores:

Francine Náthalie Ferraresi Rodrigues Queluz - Doutora, Universidade São Francisco.

Elizabeth Joan Barham - Doutora, Universidade Federal de São Carlos.

Lígia de Santis - Doutoranda, Universidade Federal de São Carlos.

Vanessa Santiago Ximenes - Mestra, Universidade Federal de São Carlos, SP, Brasil.

Acácia Aparecida Angeli dos Santos - Doutora, Universidade São Francisco, SP, Brasil.

Endereço para correspondência:

Francine Náthalie. Ferraresi Rodrigues Queluz

Rua Lúcio Pereira Peixoto, 95 apto 57 - Jardim Chapadão

13070-028 Campinas, SP, Brasil

<francine.queluz@gmail.com>

Recebido em: 11.08. 2017

Aceito em: 06.03.2018 